\title{
37 Wieder russisch?! Die Krim nach der zweiten Annexion von 2014
}

„Was geht eigentlich drunten auf der Krim vor? Ist das ukrainische Militär auf eine russische Invasion vorbereitet?“ Bixby zuckte die Achseln. „Darauf kann ich Ihnen nur eine nicht klassifizierte Antwort geben. Ich weiß, dass Sie immer noch die entsprechende Sicherheitsfreigabe haben, aber ich habe trotzdem noch nicht begriffen, was zum Teufel Sie hier wirklich wollen.“ [...] „Ich bin nur ein amerikanischer Tourist, der darüber nachdenkt, seinen Urlaub in Odessa zu verbringen.“ Bixby schüttelte den Kopf. „Okay... Also, an Ihrer Stelle würde ich lieber nach Maui gehen [...]. Die Krim wird schon bald hochgehen. Die Russen sind bereit, einzumarschieren. Sie suchen nur noch nach einem Vorwand. Die Ukrainer verlegen ihrerseits Truppen in die Region, um sie notfalls zu vertreiben [...]. Höchstwahrscheinlich werden die Russen diese ukrainischen Truppenbewegungen als Provokation bezeichnen, die mit einer Militäraktion beantwortet werden müsse.“ Mit dem Argument, die russischen Staatsbürger auf der Krim zu schützen. „Genau.“”

Dieser Dialog stammt nicht, wie man meinen könnte, aus einem Gespräch zwischen zwei US-amerikanischen Geheimdienstmitarbeitern unmittelbar vor der ab Februar 2014 beginnenden „Arbeit zur Rückholung der Krim nach Russland“2, sondern aus dem Thriller „Command Authority. Kampf um die Krim“ von Tom Clancy (1947-2013). Dieser war Schöpfer zahlreicher erfolgreicher Krimis und Erfinder der auch auf der Leinwand in der Verkörperung des Schauspielers Harrison Ford immens erfolgreichen Agentenfigur Jack Ryan. In „Command Authority“ hat es Jack Ryan bereits zum Präsidenten der Vereinigten Staaten von Amerika gebracht, und sein Gegenspieler ist der unschwer als Vladimir Putin zu erkennende Präsident der Russländischen Föderation mit Namen Valerij Volodin. Nun sind mehr oder weniger literarische Umsetzungen historischer Ereignisse nichts Besonderes, selbst für einen Autor wie Clancy, der aus dem Bereich der „Leichensackliteratur“ stamme und der „literarisch eben doch nicht mehr als ein Dritt-Liga-Le Carré“ gewesen sei, wie es ein Rezensent uncharmant in „Die Welt“ ausdrückte. ${ }^{3}$ Erstaunlich ist dennoch das Erscheinungsdatum des englischsprachigen Originals, nämlich der 3. Dezember 2013 (die deutsche Version erschien ein knappes Jahr später): Dies war Monate bevor der sog. Euromaidan richtig an Fahrt aufgenommen hatte. Die ukraineweiten Bürgerproteste richteten sich damals bekanntlich gegen die überraschende Nichtunterzeichnung des Assoziierungsabkommens mit der Europäischen Union durch den prorussischen Präsi-

1 Clancy u. Greaney, 239. Clancy arbeitete oft mit Co-Autoren zusammen, hier mit Greaney.

2 So Präsident Putin in der vom russischen Kanal „Rossija 1“ erstmalig am 15.03.2015 ausgestrahlten Sendung Krym (2017).

3 Krekeler (2014).

○ OpenAccess. ( 2020 Kerstin S. Jobst, publiziert von De Gruyter. ((c))/BY Dieses Werk ist lizenziert unter der Creative Commons Attribution 4.0 International. https://doi.org/10.1515/9783110520620-039 
denten Janukovyč sowie gegen dessen kleptokratisches Regime. Eine Entwicklung nahm damit ihren Anfang, die in die Flucht Janukovyčs nach Russland, seine letztlich nicht gesetzeskonforme Amtsenthebung durch das Parlament in Kiew (die Rada), den Beginn der Kampfhandlungen in der östlichen Ukraine und die russische Annexion der Krim mündete; dies ist ein Konflikt, der bis heute (Winter 2018) andauert und bisher schon über 10.000 Tote gefordert hat.

Clancy selbst erlebte weder das Erscheinen seines Buches noch die „Rückholung der Krim nach Russland“, denn der 1947 geborene Autor starb im Alter von nur 66 Jahren bereits im Oktober 2013. Er bewies in jedem Fall mehr Gespür für die Pläne des Kremls als die meisten ExpertInnen und politischen AnalystInnen, die vom russischen Eingreifen auf der Krim und in der Ostukraine kalt erwischt wurden. In Clancys Roman geriet die „Rückholung der Krim nach Russland“ zu einer hochbrisanten militärischen Angelegenheit - „Langstreckenraketen schalteten ukrainische Verteidigungsstellungen aus, und Kampfbomber zerstörten Flugplätze auf der östlichen Krim“, und dies, nachdem „vor einigen Monaten in Estland [...] Panzer über die Grenze nach Westen“ gerollt seien; es ist von „erbitterten Panzergefechten und dem gegenseitigen Beschuss von Grad-Mehrfachraketenwerfern“ die Rede. ${ }^{4}$ In der Realität verhielt es sich - Glück im Unglück etwas anders: Der Nato-Staat Estland wurde nicht wie im Roman von russischen Truppen überrollt, was nämlich den Bündnisfall nach Artikel 5 des NATO-Vertrages ausgelöst und vermutlich einen Krieg zwischen der Russländischen Föderation und dem westlichen Bündnis zur Folge gehabt hätte. Und auch die Okkupation der Krim gestaltete sich anders als von Clancy dargestellt, nämlich vergleichsweise gewaltarm, auch wenn der Akt als solches gewaltsam war. Dieser Akt stellt die erste Besetzung eines Territoriums eines anderen Staates durch eine Großmacht seit dem Ende des Zweiten Weltkrieges dar. Dem ukrainischen Staat, in dem es durch den Euromaidan das zweite Mal nach der sog. Orangen Revolution gelungen war, durch eine ,spontane zivilgesellschaftliche Massenbewegung“ einen Machtwechsel herbeizuführen, ${ }^{5}$ fehlten die Macht und die Möglichkeiten dies zu verhindern. Hinzu kam, dass im Osten der Ukraine, zum Teil auch im Süden, aber in jedem Fall auf der Krim die Zustimmung zu Kiew und einem ukrainischen nationalen Projekt geringer war (und ist) als etwa im Westen oder in der Zentralukraine. Eine diffuse russisch-sowjetische Einstellung, der mit über 68 Prozent russischstämmiger Menschen höchste Anteil innerhalb der Ukraine sowie die unbefriedigende sozial-ökonomische Lage, die der ukrainische Staat nicht zu lösen vermochte, hatten gerade auf der Krim dazu geführt, dass

4 Clancy u. Greaney (2014), $534 \mathrm{f}$.

5 Kappeler (2014), 346. Vgl. dort auch (338-351) zum Euromaidan. 
viele ihre Hoffnungen auf Moskau richteten. Mit dem abermaligen zivilgesellschaftlichen Aufbegehren in anderen Teilen des Landes konnten Krim-BewohnerInnen nichts anfangen. Im Gegenteil, im Verlauf des Februars 2014 fanden auf der Halbinsel mehrere prorussische Demonstrationen statt. Im Parlament der Autonomen Republik der Krim sprachen sich Abgeordnete offen für den Wiederanschluss an Russland aus. Der damalige Präsident des Krimparlaments, Vladimir A. Konstantinov ( $\left.{ }^{\star} 1956\right)$, flog sogar zu politischen Gesprächen nach Moskau ,und wurde ausgiebig mit Aussagen zitiert [...], die der Kreml jederzeit als „Hilferuf‘ interpretieren könnte“, so war am 20. Februar 2014 in der „Frankfurter Allgemeinen Zeitung“ zu lesen. ${ }^{6}$ Und das tat der Kreml dann auch.

Moskau hatten die Ereignisse in Kiew rund um die Annäherung an die EU von Anbeginn mit Sorge erfüllt, erachtete man doch die Ukraine (wie andere Teile der ehemaligen Union auch) als Teil der eigenen Einflusssphäre bzw. als (so der offizielle Begriff) „Nahes Ausland“ (russ.: Bližnee zarubeže). Und besonders ,nah“ fühlen sich viele RussInnen den UkrainerInnen, deren nationalen Sonderweg viele ,GroßrussInnen“ mit Unverständnis zur Kenntnis nahmen und nehmen. Sowohl die Orange Revolution als auch der Euromaidan ließen den Kreml fürchten, die Ukraine nun endgültig zu verlieren. Nachdem die Olympischen Winterspiele in Soči am 23. Februar 2014 beendet waren und sich die Lage in der Ukraine nicht im Sinne Moskaus beruhigt hatte, wurde am 27. Februar eingegriffen: Gutausgebildete und schwerbewaffnete Kämpfer ohne Hoheits- und Rangabzeichen besetzten wichtige strategische Einrichtungen auf der Krim wie den Flughafen in Simferopol', Standorte der ukrainischen Armee und das Parlament. Diese waren im Sinne des Kriegsvölkerrechts keine Kombattanten und wurden wahlweise als „kleine grüne Männer“ - ukrainischerseits (ukr.: zeleni čolovički) oder als „freundliche Leute“ (russ.: vežlivye ljudi) bezeichnet. In der Tat handelte es sich aber um Angehörige des russischen Militärs und seiner Spezialeinheiten, die durch die legal auf der Krim stationierten Soldaten ergänzt wurden. Sie wurden dann bald von einer nicht unerheblichen Zahl eigentlich in ukrainischen Diensten stehender Truppen verstärkt. ${ }^{7}$ Sergej V. Aksenov ( $\left.{ }^{\star} 1972\right)$, der seit 2010 einer weitgehend einflusslosen Partei mit Namen „Russische Einheit“ (russ.: Russkoe Edinstvo) vorgestanden hatte, wurde zeitgleich von einigen Abgeordneten im militärisch besetzten Parlament in Simferopol' zum Präsidenten erklärt, was nicht in deren Befugnis lag. Mittlerweile ist er Mitglied in der alles dominierenden Partei „Einiges Russland“ (russ.: Edinaja Rossija) und Präsident der Republik

6 Schmidt (2014).

7 Kappeler (2014), 352, geht davon aus, dass sich mehr als die Hälfte der auf der Krim befindlichen ukrainischen Soldaten bis zum 24.03.2014 in russische Dienste gestellt haben. 
Krim innerhalb der Russländischen Föderation. ${ }^{8}$ Seine ,Regierung‘ rief sogleich Moskau um „Schutz vor gewaltbereiten ukrainischen Nationalisten und Extremisten“ an;” in bewährter sowjetischer Manier (u.a. Ungarn 1956, ČSSR 1968, Afghanistan 1979) gewährte Moskau diese ,Hilfe‘. Am 16. März wurde ein schnell anberaumtes Referendum abgehalten, in dem angeblich über 90 Prozent der zur Abstimmung gegangenen Krim-BewohnerInnen - nach offiziellen Zahlen 82 Prozent aller Wahlberechtigten - für den Anschluss an Russland plädierten; diese Abstimmung war, was selbst das russischsprachige Wikipedia nicht verschweigt, nicht mit der immer noch geltenden ukrainischen Verfassung in Einklang zu bringen. ${ }^{10}$ Einen Tag später wurde die Unabhängigkeit der Krim von der Ukraine erklärt und am 18. März zwischen Putin und Vertretern der illegitimen Krim-Regierung ein Vertrag über die Aufnahme der Halbinsel in die Russländische Föderation unterzeichnet. Genau genommen traten zwei neue sog. Unionssubjekte in diese ein - die Republik Krim und die Stadt föderalen Ranges, Sevastopol', womit sich die besondere Bedeutung der ,Heldenstadt' abermals auch administrativ abbildete.

Bemerkenswert ist die Rede, die Präsident Putin anlässlich der dann auch offiziell zum Abschluss gebrachten Annexion - oder, wie Putin sagte, der „Wiedervereinigung“ - vor dem Föderationsrat und der Staatsduma am 18. März 2014 hielt: Einleitend zeigte er sich von der Rechtmäßigkeit der Abstimmung überzeugt, sie habe internationalen Rechtsnormen entsprochen; dies war allerdings eine Auffassung, die außerhalb Russlands nur von wenigen Fachleuten geteilt wurde. ${ }^{11}$ Putin bediente sich in dieser Rede nicht das erste und nicht das letzte Mal ausgiebig aus dem Repertoire historischer Legitimationen für eine russische Krim (Kapitel 2), sei doch dort „buchstäblich alles von unserer gemeinsamen Geschichte und unserem Stolz erfüllt.“ Die Halbinsel sei der Ort der Taufe der Rus’, sie sei heilig und voller Symbole des russischen militärischen Ruhms und Heldenmutes. ${ }^{12}$ Den traditionellen Vielvölkercharakter der Halbinsel - „Russen und Ukrainer, Krimtataren und Vertreter anderer Völker lebten und arbeiteten nebeneinander“ - verschwieg er nicht, machte aber deutlich, dass RussInnen die absolute Mehrheit unter den ca. 2,2 Millionen BewohnerInnen stellten. Die 350.000 ethnischen UkrainerInnen seien zudem russophon und selbst die krimtatarische Bevölkerung sei „auf Russland ausgerichtet“. Davon konnte allerdings

8 Für eine Annäherung an die Person Aksenovs vgl. Shuster (2014).

9 Kiew (2014).

10 Krymskij krizis (2018).

11 Zur Bewertung der Aneignung der Krim 2014 durch internationale Rechtsgelehrte vgl. Luchterhandt (2014).

12 Hier und im Folgenden: Obraščenie (2014). 
nicht die Rede sein, hatten die meisten KrimtatarInnen das Referendum doch boykottiert und sich klar gegen die Annexion positioniert. Die stalinistischen Deportationen wurden von Putin in recht verklausulierter Form erwähnt: Die krimtatarische Bevölkerung habe, „wie auch einige andere Völker der UdSSR, grausame Ungerechtigkeiten“ erfahren, das größte Opfer aber hätte das russische Volk erbracht. Als ein Angebot des Präsidenten an die muslimischen und die ukrainischen KrimbewohnerInnen mag die Offerte der Einführung dreier gleichberechtigter Sprachen auf der Krim gelten; neben dem Russischen zählten dazu das Ukrainische, welches selbst vor der Annexion dort ein Schattendasein gefristet hatte, und eben auch das Krimtatarische; dies war allerdings bereits vor 2014 der Fall gewesen. Die Übergabe der Krim an die Ukraine 1954 und deren Verbleib bei dieser nach 1991 wurde von Putin - kaum erstaunlich - als ungesetzlich bezeichnet. Die Halbinsel sei „von Hand zu Hand gegeben worden wie ein Sack Kartoffeln“ („kak mešok kartoški“), was eine krasse historische Ungerechtigkeit gewesen sei, wogegen Russland aber seinerzeit nichts habe ausrichten können. Die seiner Meinung nach chaotische Lage in der Ukraine durch den, wie er es nannte, „Putsch“ (= Euromaidan) habe die russischsprachigen Krim-BewohnerInnen bewogen, Moskau um den Schutz ihrer Rechte und ihres Lebens zu bitten... „Natürlich konnten wir nicht anders, als auf diese Anfrage zu antworten“, so Putin. Der Präsident der Russländischen Föderation versprach bzw. drohte zudem, dass Russland die Interessen der „Millionen russischsprachiger Bürger [...] in der Ukraine [...] immer mit politischen, diplomatischen und rechtlichen Mitteln vertreten“ werde.

Die von Moskau beanspruchte Schutzfunktion russischer Menschen im sog. Nahen Ausland hatte sich nach dem Zerfall der Sowjetunion erstmalig im August 2008 während des Georgien-Krieges um Süd-Ossetien und Abchasien materialisiert. Der Thriller-Autor Clancy hatte offenbar diesen Fall vor Augen und erklärte Russlands Vorgehen in seinem Roman wie folgt:

„Sie wissen wahrscheinlich, dass diese ethnischen Russen nur deshalb zu russischen Staatsbürgern wurden, weil Moskau Pässe an die Ukrainer russischer Abstammung verteilt hat. Es war von Anfang bis Ende eine FSB-Operation [FSB=Federal'naja služba bezopasnosti Rossijskoj Federacii (Föderaler Sicherheitsdienst der Russländischen Föderation); KSJ], die den russischen Einmarsch vorbereiten sollte [...]. Schließlich werden sie erklären: ,Wir müssen unseren Staatsbürgern auf der Krim zu Hilfe kommen'. Sie haben dasselbe vor ein paar Jahren in Georgien gemacht. “13.

13 Clancy u. Greaney (2014), 239 f. 
Tatsächlich hatte die 2008 amtierende ukrainische Administration unter Juščenko Doppelstaatsbürgerschaften für die BürgerInnen der Ukraine aus diesem Grunde unter Strafe gestellt. ${ }^{14} 2014$ argumentierten Putin und andere etwas anders, da sie zumeist von der russophonen Bevölkerung in der Ukraine sprachen, die zu schützen sei. Damit beanspruchte Moskau sogar noch mehr, ging es doch nicht mehr allein um den angeblichen Schutz ethnischer RussInnen. Russischerseits hatte es übrigens nach der Georgien-Krise eine bedeutende formale Anpassung zur Verwirklichung der Ziele gegenüber dem „Nahen Ausland“ gegeben: die unter Präsident Dmitrij A. Medvedev verabschiedete - und deshalb manchmal auch nach ihm benannte - „Militärdoktrin 2020“ von 2010. Neben vielen anderen Punkten erweiterte diese auch die Einsatzoptionen der russländischen Streitkräfte im Ausland, u. a. zum ,Schutz' der Bürgerinnen und Bürger Russlands, die in einer der ehemaligen Sowjetrepubliken lebten. Auf diese Art und Weise hatte der Kreml nachträglich versucht, das Vorgehen in Georgien zu rechtfertigen. ${ }^{15}$

Nicht nur in der zitierten Rede Putins wird immer wieder von einer Bedrohung nationaler Minderheiten - hier der RussInnnen in der Ukraine - gesprochen und damit die Annexion quasi als ein Akt der Selbstverteidigung dargestellt. Nun ist im kodifizierten Völkerrecht nationale Selbstbestimmung durchaus ein hohes Gut, welches allerdings in realiter häufig mit einem wohl noch höher erachteten kollidiert - dem der territorialen Integrität von Staaten. Im Falle der Krim-Annexion wurde die der Ukraine in jedem Fall verletzt, soviel ist festzuhalten. Und benötigten russische Menschen auf der Halbinsel den Schutz Moskaus, wurden sie drangsaliert oder bedroht? Das war „überhaupt nicht erkennbar“, so der Völkerrechtler Bruno Simma, ehemaliger Richter am Internationalen Gerichtshof in Den Haag und Professor für Völkerrecht an der Ludwig-Maximilians-Universität München, der die russische Intervention verurteilt. Zugleich beklagt er, dass das völkerrechtliche Gewalt- und Interventionsverbot in der jüngeren Vergangenheit auch von den USA oder Teilen der Europäischen Union, z. B. im Falle des Kosovo (auf den sich die russische Seite gerne berief), „immer wieder durchlöchert und aufgeweicht worden“ sei. ${ }^{16}$ Die Krim-Annexion wird recht übereinstimmend von ExpertInnen als Verstoß der Russländischen Föderation gegen das allgemeine Gewaltverbot des Artikels 2, Nr. 4 der Charta der Vereinten Nationen gewertet. Darin heißt es:

14 Jobst (2015b), $260 \mathrm{f}$.

15 Im Überblick: Klein M. (2010).

16 Hipp (2014). 
Alle Mitglieder unterlassen in ihren internationalen Beziehungen jede gegen die territoriale Unversehrtheit oder die politische Unabhängigkeit eines Staates gerichtete oder sonst mit den Zielen der Vereinten Nationen unvereinbare Androhung oder Anwendung von Gewalt. ${ }^{17}$

Es gibt nur wenige russische JuristInnen, die dieser Argumentation folgen. ${ }^{18}$ Auch wenn einzelne Politiker wie der ehemalige SPD-Vorsitzende Matthias Platzeck ( $\left.{ }^{\star} 1953\right)$ oder auch der hochgeschätzte Altbundeskanzler der Bundesrepublik Deutschland, Helmut Schmidt (1918-2015), großes Verständnis für den Kurs Putins gegenüber der Krim und der Ostukraine zeigten, ${ }^{19}$ so reagierte u.a. die Europäische Union mit umfangreichen Sanktionen gegenüber der Russländischen Föderation. Bis heute (d.h. Ende 2018) wurden diese immer wieder verlängert. Während gegenwärtig die ,russische Krim‘ ein Faktum ist, dauern die militärischen Auseinandersetzungen in der Ostukraine an. Wann und ob Moskau den hybriden Krieg gegen die Ukraine, deren sukzessive Destabilisierung geplant ist, einstellen wird, ist nicht vorauszusehen. Tatsache ist, dass die großen Hoffnungen, die viele Ukrainerinnen und Ukrainer in den Euromaidan und die Regierung Petro Porošenkos ( $\left.{ }^{\star} 1965\right)$ gesetzt hatten, sich bislang nicht erfüllt haben.

Dies gilt nicht zuletzt für die KrimtatarInnen, die sich in ihrer überwältigenden Mehrheit für den Verbleib der Krim bei der Ukraine ausgesprochen haben. Die neuerliche Eingliederung der Krim in ein russisches Staatswesen, welches im kollektiven Gedächtnis für diese negativ besetzt ist, stellt sie vor weitere Herausforderungen. Der abermalige Verlust ihrer Heimat wird befürchtet und ist für manche von ihnen schon wieder Realität geworden. Einer von diesen ist ausgerechtet der 1998 als prowestlicher Abgeordneter ins ukrainische Parlament gewählte krimtatarische Nationalheld Cemilev (Kapitel 35). Ihm und Refat Çubarov wurde russischerseits die Einreise auf die Krim für die nächsten fünf Jahre verweigert. Weitere Pressionen der tatarischen Bevölkerung folgten, die aber eher als eine ,Gleichschaltung' an die insgesamt problematische politische und zivilgesellschaftliche Situation in der Russländischen Föderation denn als eine genuin antitatarische Maßnahme interpretiert werden sollten. Nach dem sog. Referendum befürchteten krimtatarische Vertreter zu Recht Konflikte mit der neuen KrimRegierung unter der Führung Aksenovs und erfolgstrunkenen russischen Nationalisten. Auch deshalb verließen, so die Berechnungen der „Gesellschaft für bedrohte Völker“, von den rund 280.000 KrimtatarInnen unmittelbar nach der russischen Machtübernahme mehrere Tausend die Halbinsel. ${ }^{20}$ Hunderte retteten

17 Charta (2018).

18 Gall (2015).

19 Russland-Politik (2014); Krim-Krise (2014).

20 Gesellschaft für bedrohte Völker (2017), 7. 
sich beispielweise in das westukrainische L'viv (ukr.; poln.: Lwów; dt.: Lemberg), obgleich tatarische Akteure zum Bleiben auf der Halbinsel aufriefen. ${ }^{21}$ Der Qurultay (Kapitel 36) sprach sich für Gewaltlosigkeit und eine partielle Kooperation mit der russischen Macht aus und hoffte mit einer gewissen Naivität auf die Errichtung einer national-territorialen Autonomie für ihre Nationalität. Zugleich erklärte er das Referendum aber für illegal und suchte die Verbindung zu internationalen Organisationen (Vereinte Nationen, Europarat, OSZE, Organisation Islamischer Zusammenarbeit). ${ }^{22}$ Die Türkei, die ja eine enge historische, kulturelle und auch sprachliche Verbindung zur krimtatarischen Nationalität besitzt und wo zudem eine große krimtatarische Diaspora lebt, engagierte sich erstaunlich wenig für die Glaubensbrüder und -schwestern, wohl um die Beziehungen zu Moskau nicht zu gefährden. ${ }^{23}$

Die russische Führung signalisierte - wie bereits am Beispiel der Rede Putins vom 18. März 2014 gezeigt wurde - anfänglich ein gewisses Entgegenkommen gegenüber der krimtatarischen Bewegung. Doch immer wieder kommt es auf der Halbinsel zu Zusammenstößen zwischen dieser, russischen Milizen sowie offiziellen Stellen; ein vertrauensvolles Miteinander konnte sich schon aufgrund der historischen Erfahrungen kaum ausbilden. Fälle von „Verschwindenlassen“, Entführungen und Prozesse mit zweifelhaften juristischen Standards gegen politisch aktive KrimtatarInnen sind häufig. ${ }^{24}$ Auch in westlichen Medien, die ihr Interesse am Krim-Thema schnell wieder verloren hatten, machte der Fall der Schließung des auch in krimtatarischer Sprache ausstrahlenden TV-Senders ATR Anfang 2015 Furore. Der Sender gehört dem krimtatarischen Geschäftsmann Lenur Edem oğlu İslâmov (*1966) und hatte sich gegen die russische Annexion in Stellung gebracht. Mehrere Male wurde der Sender von der Polizei durchsucht und schließlich endgültig geschlossen, da er angeblich nicht ordnungsgemäß registriert war. ${ }^{25}$ Mittlerweile sendet er aus der nicht-besetzten Ukraine weiter.

Durchsuchungen und Repressionen vergegenwärtigten auch der Meclis (Kapitel 36), die Wochenzeitung „Avdet“ und andere krimtatarische Einrichtungen. ${ }^{26}$ Dem Meclis wurde russischerseits politisch-religiöser Extremismus vorgeworfen oder sogar dem „Islamischen Staat“ nahezustehen. Seit dem 26. April 2016 ist er deshalb verboten. Getreu dem Motto „Teile und Herrsche“ hatte Moskau prorus-

21 Eichhofer (2014).

22 Halbach (2014).

23 Zur Haltung der Türkei in der Krim-Krise vgl. Gasimov (2014).

24 Vgl. Gesellschaft für bedrohte Völker (2017), $7 \mathrm{f}$.

25 Krymskij telekanal (2015).

26 Ich folge Malek (2017), 192-196, und Halbach (2014). 
sische Alternativorgane wie die „Qurm birligi“ („Einigkeit der Krim“) gebildet. ${ }^{27}$ Ohnehin gibt es einzelne krimtatarische Politiker sowie die hohe Geistlichkeit, die sich mit der russischen Macht arrangiert haben. ${ }^{28}$ Uwe Halbach, Experte für die muslimischen Regionen im (post-)sowjetischen Raum, hat Ende 2014 auf die aus der Annexion erwachsenen ethno-politischen Herausforderungen zwischen den russischen, ukrainischen und tatarischen Bevölkerungsgruppen hingewiesen. Er sieht zudem das Risiko, dass sich „,in Russland und in anderen Teilen des postsowjetischen Raums tätige islamistische Netzwerke der krimtatarischen Frage annehmen - unter der Parole des Kampfs gegen die Unterdrückung von Muslimen. [...] Russland [läuft] mit seinem Krim-Anschluss womöglich Gefahr, sich eine neue islamistische Front zu eröffnen.“ Und einige krimtatarische Krieger hätten sich bereits nach Syrien abgesetzt. ${ }^{29}$

Weitaus mehr Krimtataren kämpfen allerdings in den ukrainischen Streitkräften, auch in der umkämpften Ostukraine sowie in einer 2015 gebildeten Freiwilligen-Einheit. Das „Noman Çelebicihan Batalyonı“ ist nach dem Aktivisten des Ersten Weltkriegs und Schöpfer der inoffiziellen krimtatarischen Hymne Noman Çelebicihan (Kapitel 31) benannt, der im Bürgerkrieg von den Bol’ševiki hingerichtet wurde. Organisiert wurde es von dem bereits erwähnten İslâmov, stationiert ist es im Gebiet von Cherson westlich der Krim. Die gut 500 Mann umfassende Gruppe plant, so İslâmov Ende 2015, „to defend the borders of the Crimea inside Crimea“30; zur Vertreibung Russlands von der Halbinsel ist es bisher allerdings nicht gekommen.

Im Gegenteil werden die Verbindungen zwischen der Krim und Russland in vieler Hinsicht immer enger. Wie bereits 1783, so machte sich die russische Administration auch 2014 daran, die Krim ,zügig in den russischen Staat [zu] integrieren“31 und die Verbindungen zwischen dem Festland und der Halbinsel zu festigen. Dies ist auch ganz wörtlich zu nehmen, denn bis vor kurzem fehlten Verbindungswege, um die Krim von Russland aus zu versorgen, sah man von den Fährverbindungen in der Straße von Kerč' einmal ab. Viele Waren und auch Energie mussten von Norden über die Landzunge von Perekop und damit von der Ukraine aus eingeführt werden. Und die ukrainische Seite ließ häufiger ihren einzigen ,Muskel' spielen, den sie gegenüber Moskau in der Krim-Frage hat, indem sie die Grenze immer wieder mal schloss. Der Bau einer „patriotischen Brücke“ zwischen Kerč’ und Taman war in Moskau schnell beschlossen; Pläne

27 Rubljow (2017).

28 Malek (2017), 196 f.

29 Halbach (2014).

30 Crimea blockade (2017).

31 Kappeler (2014), 354. 
dafür lagen schon länger vor ${ }^{32}$ - und wurden tatsächlich umgesetzt. Die ca. neunzehn Kilometer lange Brücke (russ.: Krymskij most) wurde im Mai 2018 in einer ungeheuren Schnelligkeit gebaut und für den Straßenverkehr geöffnet. In einer wohlinszenierten Zeremonie wurde sie von einem sichtlich stolzen Präsidenten Putin eröffnet, der persönlich einen Lastkraftwagen über die Brücke steuerte. Er demonstrierte damit der Welt nicht nur die russische Ingenieurskunst, sondern auch, dass er einen LKW lenken kann. 2019 soll die Brücke ebenso für den Eisenbahnverkehr geöffnet werden. Die ökologischen und ökonomischen Folgen des Bauwerks können noch nicht abgesehen werden. ${ }^{33}$ In jedem Fall hat Moskau mit der Krim-Brücke bessere Möglichkeiten zur Kontrolle der Straße von Kerč' und des Azovschen Meeres. An diesem liegen mit Berdjans'k und Mariupol', welches ein wichtiger Tiefseehafen für die Ukraine ist, zwei für die Ukraine wichtige Umschlagplätze. In Mariupol' konzentriert sich überdies die wichtige ukrainische Stahlindustrie. Kiew war nicht nur aus politischen, sondern auch aus wirtschaftlichen Gründen unter den gegenwärtigen Umständen gegen den Brückenbau: Großschiffe über 33 Meter können diese nicht durchfahren, immer häufiger wurden russischerseits aufwendige und teure Kontrollen durchgeführt, obwohl 2003 vereinbart worden war, dass beide Staaten dieses Meer befahren dürften. Am 25. November $2018 \mathrm{kam}$ es dann zu einem schwerwiegenden Zwischenfall, der die Bedeutung der Krim als ein in den letzten Jahren zwar weithin vergessener, aber virulenter Krisenherd vor Augen führte: Die russische Marine brachte drei Schiffe der ukrainischen Marine unter ihre Kontrolle, eines von ihnen soll vordem gerammt worden sein, und verhaftete die Besatzung, wobei es Verletzte gab. Russland sprach von einer Provokation Kiews, hätten die ukrainischen Schiffe doch die Grenze zwischen der Ukraine und Russland illegal überquert. ${ }^{34}$ Der ukrainische Präsident Petro Porošenko ließ daraufhin erstmalig seit dem Ausbrechen des Konflikts 2014 das Kriegsrecht ausrufen; allerdings beschnitt die Rada dessen zeitlichen und inhaltlichen Umfang erheblich, da der Verdacht doch nicht von der Hand zu weisen war, dass der angeschlagene Präsident sich vor den bevorstehenden Wahlen im Frühjahr 2019 noch einmal als ,harter Hund“ prä-

32 Birger (2015). Bereits vor dem Ersten Weltkrieg wurde der Bau einer Brücke diskutiert. Im Zweiten Weltkrieg versuchten sich sowohl deutsche und später dann auch sowjetische Akteure daran. Die deutsche Anlage wurde 1943 von der Wehrmacht gesprengt, die eilig errichte sowjetische brach im Februar 1944 unter dem Druck von Eismassen zusammen. Vgl. u. a. Hoppe (2016). 33 Romashchenko u.a. (2018).

34 Esch (2018). 


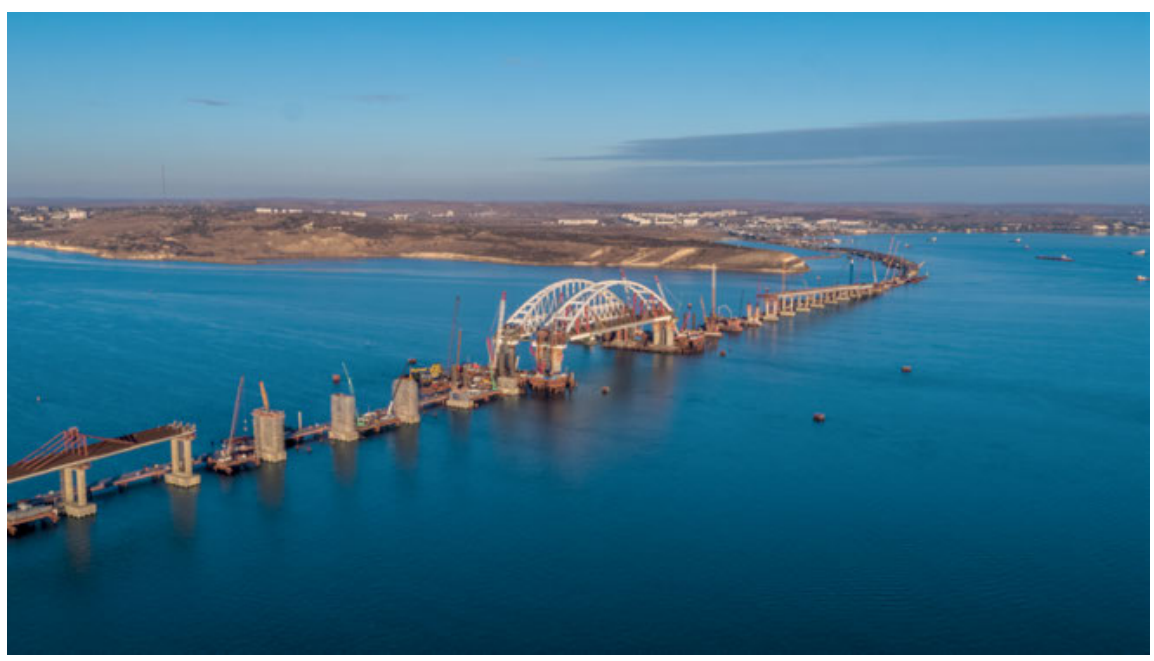

Abb. 16: Bau der Krim-Brücke, 13. Oktober 2017

sentieren wollte. ${ }^{35}$ Die Wahl des neuen Präsidenten Volodymyr Zelenskyj (*1978) im Mai 2019 zeigte, dass diese Strategie beim Wahlvolk nicht verfing. ${ }^{36}$

Kiew hat zwar immer wieder die russische Politik etwa gegenüber den KrimtatarInnen kritisiert - und zwar viel heftiger und schneller als es die EU dann letztlich tat. ${ }^{37}$ Dennoch konnte man sich bis zur Festsetzung der ukrainischen Marineboote nicht des Eindrucks erwehren, dass Kiew die Krim verloren gegeben hat und den drohenden endgültigen Verlust der Ostukraine als deutlich gravierender betrachtet.

Ist die Krim nun wirklich russisch geworden, also „Krym naš“, wie die Parole seit 1991 immer wieder lautete? Gehört sie Russland? Der bereits zitierte Halbach antwortet darauf mit einem bedenkenswerten Vergleich: „In Wirklichkeit gehört

35 Zum Ablauf vgl. u.a. Ballin (2018). Vgl. auch den Beitrag Stomporowski (2018). Die Autorin macht „Vorverurteilungen“ der russischen Seite bei deutschen Politikern und Medien aus; der insgesamt gut recherchierte und kenntnisreiche, aber eindeutig um Verständnis für Russland werbende Artikel beklagt auch die kritische Haltung der Partei „Die Grünen“ gegenüber Moskau sowie das Wirken der NATO in der Region.

36 Welche Strategie Zelens'kyj letztlich in der Krim-Frage verfolgen wird, ist noch nicht erkennbar, er hat allerdings wiederholt den Dialog mit Russland angeboten, ohne von der Forderung nach Rückgabe der Krim abzuweichen.

37 Krim (2016). 
diese Region so selbstverständlich zu Russland wie Algerien zu Frankreich gehört hat - nämlich kolonialgeschichtlich." ${ }^{\text {38 }}$

38 Halbach (2014). 